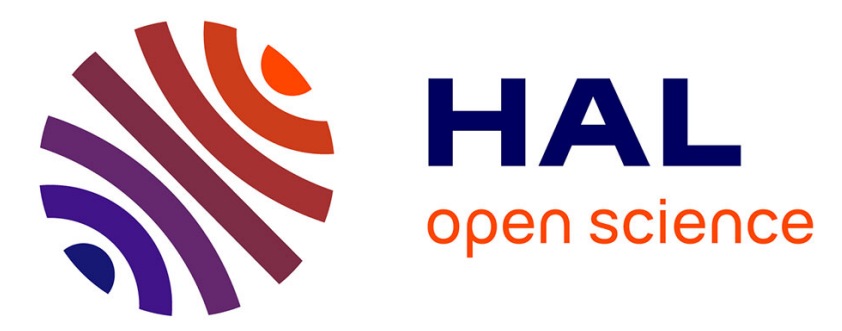

\title{
CARACTÉRISATION DU MILIEU MARIN COMME CANAL DE TRANSMISSION HORIZONTALE
}

\author{
G. Loubet, F. Vial
}

\section{To cite this version:}

G. Loubet, F. Vial. CARACTÉRISATION DU MILIEU MARIN COMME CANAL DE TRANSMISSION HORIZONTALE. Journal de Physique IV Proceedings, 1992, 02 (C1), pp.C1-937-C1-940. 10.1051/jp4:19921204. jpa-00251170

\section{HAL Id: jpa-00251170 https://hal.science/jpa-00251170}

Submitted on 1 Jan 1992

HAL is a multi-disciplinary open access archive for the deposit and dissemination of scientific research documents, whether they are published or not. The documents may come from teaching and research institutions in France or abroad, or from public or private research centers.
L'archive ouverte pluridisciplinaire HAL, est destinée au dépôt et à la diffusion de documents scientifiques de niveau recherche, publiés ou non, émanant des établissements d'enseignement et de recherche français ou étrangers, des laboratoires publics ou privés. 


\title{
CARACTERISATION DU MILIEU MARIN COMME CANAL DE TRANSMISSION HORIZONTALE
}

\author{
G. LOUBET et F. VIAL \\ Laboratoire CEPHAG - ENSIEG - CNRS URA 346, Domaine Universitaire, BP. 46, \\ F-38402 St Martin d'Hères cedex, France
}

\begin{abstract}
Résumé - A partir de nombreux résultats déja obtenus au laboratoire et d'une expérimentation récente en Méditerranée, nous présentons un essai de caractérisation du milieu marin en tant que canal de communication acoustique horizontale. Il nous semble que l'on peut distinguer deux domaines: celui des transmissions courte ou moyenne distance (entre $1 \mathrm{et} 15 \mathrm{~km}$ ) où interviennent de nombreux paramètres liés à la physique du milieu (courbe de célérité du son mais aussi influence des courants, de l'état de la mer et des fonds, de la houle...), aux conditions de transmission (profondeur d'émission, directivité et inclinaison de l'antenne...). Le 2ème domaine concerne les communications longue distance $(>15 \mathrm{~km})$ pour lesquelles le phénomène de réfraction des rayons est primordial. Cest pourquoi les transmissions courte distance sont plus délicates (essentiellement à cause d'interférences), le cas le plus défavorable étant celui des petits fonds.
\end{abstract}

\begin{abstract}
From many results already obtained in our laboratory and a recent experiment in the Mediterranean Sea, we try to characterise the underwater medium as a horizontal acoustic communication channel. We can distinguish 2 fields. The first one deals with short or middle range transmission (between 1 and $15 \mathrm{~km}$ ) in which many parameters must be considered: those connected to the physics of the medium (sound celerity graph, influence of the current, the sea state, the swell...) and to the transmission conditions: emission depth, directivity and angle of the antenna... The second one deals with long range communication $(>15 \mathrm{~km})$ which depends primarily on the ray-refraction phenomenon. Therefore, short range transmissions are more critical (essentially due to interferences); the worst case is the shallows one.
\end{abstract}

\section{INTRODUCTION}

Les transmissions acoustiques sous-marines font l'objet d'un intérêt grandissant depuis quelques années (nombreux projets européens par exemple), aussi la caractérisation du milieu marin comme canal de communication horizontale est d'une importance primordiale pour le choix des signaux à émettre, du type de modulation et de bien d'autres paramètres comme les profondeurs d'émission et réception, les méthodes de synchronisation, de suivi de porteuse... Nous n'aborderons pas le cas des transmissions verticales qui sont très particulier: faibles distances de propagation, fréquences élevées $(>50 \mathrm{kHz})$, importance de la directivité de l'antenne... ni le cas des transmissions à très courte distance $(<1 \mathrm{~km})$ qui sont du même genre. Elles font appel à des techniques de communication du type égalisation pour atteindre un fort débit.

Il est assez illusoire de vouloir caractériser le canal acoustique marin horizontal à cause du trop grand nombre de paramètres qui interviennent. Toutefois il nous semble que l'on peut distinguer grossièrement deux domaines:

* celui de communications courte ou moyenne distance (entre 1 et $15 \mathrm{~km}$ ) où interviennent de nombreux paramètres propres au type précis de transmission: directivité de l'antenne émettrice, profondeur du chenal, état de la mer et des fonds, position des émetteurs et récepteurs, influence des courants voire de la houle...

* Le deuxième concerne les transmissions longue distance $(>15 \mathrm{~km})$ pour lesquelles les paramètres classiques (pertes énergétiques, phénomène de réfraction des rayons en fonction du profil de célérité du son dans l'eau) sont primordiales. 
Pour souligner cette dichotomie, nous allons présenter les résultats d'une expérimentation récente en Méditerranée effectuée à différentes distances de propagation.

\section{TRANSMISSION COURTE OU MOYENNE DISTANCE}

Les caractéristiques précises du milieu interviennent pour ce type de transmission. Le cas le plus défavorable est celui de communication par petits fonds. Une expérimentation en Mer du Nord a déja été présentée (cf [1],[2]). Le canal, profond de $500 \mathrm{~m}$, engendrait sur $4 \mathrm{~km}$ de nombreux trajets réfléchis fondsurface. Le problème vient de ce que de nombreux trajets ont des temps de propagation et des énergies voisins. Leurs fluctuations (en temps de propagation et en amplitude), dues aux réflections de surface, bien que relativement faibles, génèrent des combinaisons variables de trajets, des interférences qui peuvent provoquer d'énormes variations de l'énergie reçue à certains instants (voire sa disparition), sur une échelle de temps de l'ordre de la seconde. Ce genre de milieu se rencontre en Mer du Nord, en Mer d'Irlande, en Baltique par exemple. Il faut éliminer le plus possible les interférences en utilisant un émetteur très directif. Un traitement d'antenne est pour ce type de communication quasiment indispensable. Bien que moins marqué que pour les petits fonds, ce phénomène d'interférences, lié à la longueur d'onde des signaux, est d'autant plus marqué que la fréquence est élevée. Il se rencontre très souvent lors de transmissions courte et moyenne distance comme nous allons le voir ci-dessous.

\section{Conditions de l'expérimentation:}

C'est une transmission horizontale, en Méditerranée, entre un bateau émetteur en station pseudo-fixe et une antenne verticale fixe comportant 6 hydrophones répartis à différentes profondeurs. Nous allons présenter les résultats pour une distance de propagation d'environ $9 \mathrm{~km}(5$ miles nautiques) et une profondeur d'émission de $160 \mathrm{~m}$.

Pour identifier le milieu, nous avons émis des signaux modulés en phase à deux états (BPSK) par des séquences binaires de longueur maximale (SBLM) émises de façon récurrente. Ces signaux sont caractérisés par une fonction d'ambiguitté présentant une très bonne résolution temporelle et fréquentielle. Ils sont d'une utilisation aisée et, à notre connaissance, ce sont les signaux les plus performants parmi les signaux à deux états de phase. Nous avons employé des SBLM de BT 511 et 1023 de "bande" $\pm 500 \mathrm{~Hz}$ et $\pm 1000 \mathrm{~Hz}$ respectivement autour d'une porteuse de $2 \mathrm{kHz}$.

Les variations de la température de l'eau en fonction de la profondeur étaient relevées sur le bateau émetteur. Nous donnons figure 1 les courbes déduites de variation de la célérité du son et le tracé de rayon correspondant pour une profi
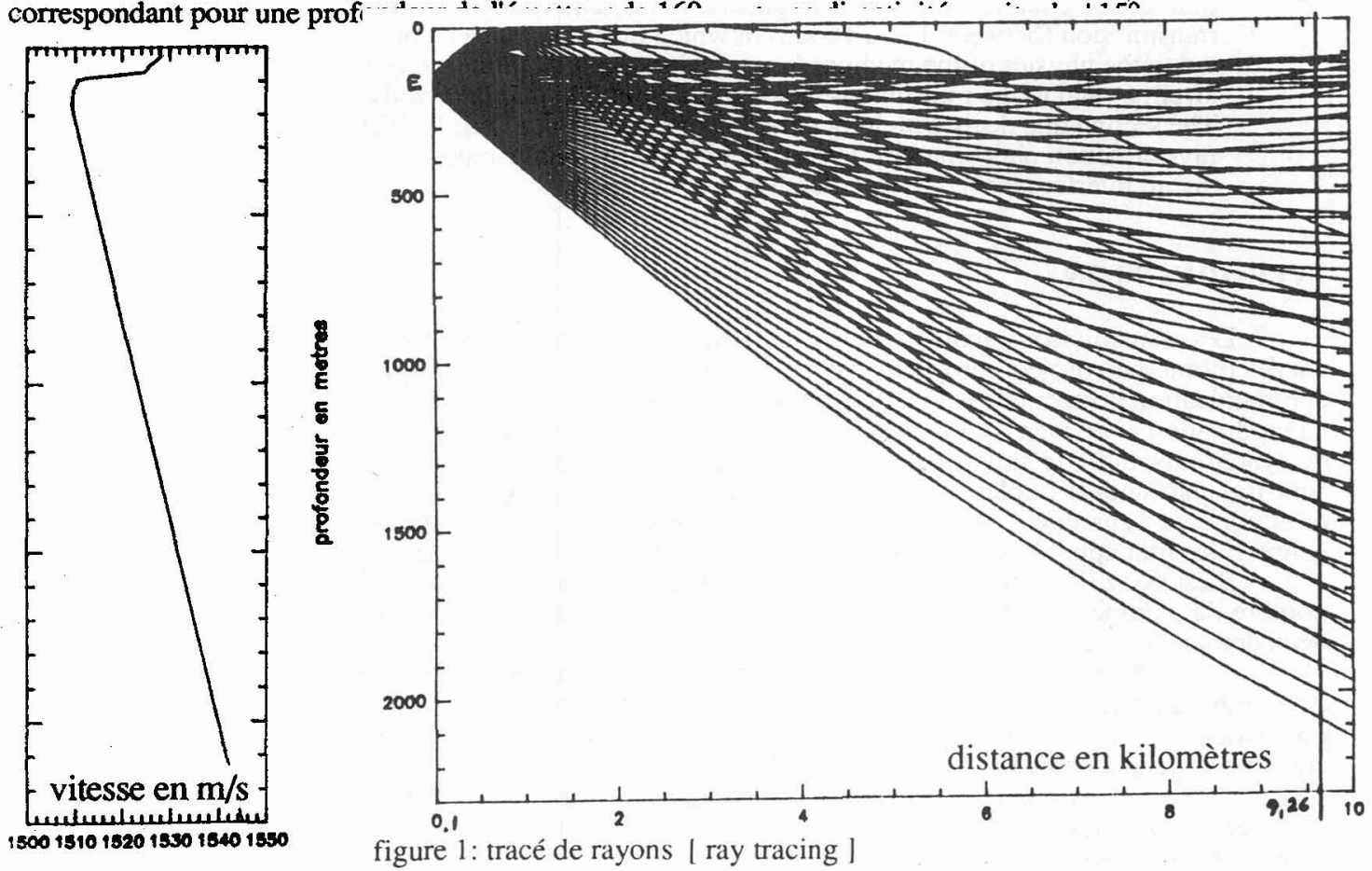


\section{Ecarts Doppler}

Nous avons mesuré par interambiguitté entre le signal reçu et des copies dopplérisées du signal émis les variations de la fréquence porteuse. Celles-ci sont de l'ordre de quelques dixièmes de $\mathrm{Hz}$ et représentent les fluctuations de la vitesse radiale du bateau dues au courant qui était de l'ordre de 1 noeud. Elles sont faibles. De manière générale, nous avons toujours relevé un élargissement fréquentiel (du aux courants, à la réverbération) de cet ordre en acoustique sous-marine. Ce phénomène est donc très peu gênant pour une communication. Par contre, si les bateaux étaient en mouvement, le décalage Doppler devrait être compensé (par interambiguité -méthode lourde- ou une technique de suivi de fréquence). Mais, pour une modélisation du milieu marin, on peut négliger l'élargissement fréquentiel [1].

\section{Aspects énergétiques}

La figure 1 indique que les capteurs peu profonds ne peuvent recevoir de l'énergie, ce que confirme l'expérimentation. En dessous d'une centaine de mètres, la transmission est comparable sur tous les capteurs, même proches du fond. L'énergie est essentiellement regroupée en un instant d'arrivée. Par contre, elle fluctue énormément, ce qui est imprévisible par tracé de rayons et le rapport signal à bruit est peu estimable. Ces anomalies présentent deux formes: des variations rapides, par moments très nettement périodiques (figure 2), qui semblent révéler l'influence de la houle (période \# 12 s double de celle de la houle) et des variations lentes (à léchelle de 5 à $10 \mathrm{mn}$ ) et très importantes (>20 dB).

Ces dernières peuvent s'expliquer par l'inclinaison de l'émetteur. Celui-ci est assez directif (-3dB d'atténuation pour un angle de $\left.\pm 7,5^{\circ}\right)$. Un tracé de rayons montre que si l'on fait varier l'angle de directivité en site de $\pm 10^{\circ}$, les rayons arrivant sur l'antenre réceptrice peuvent disparaitre. Ce phénomène est du au fait que la profondeur d'immersion est voisine de celle correspondant à la célérité minimale (figure 1). Or une inclinaison de l'émetteur de $\pm 10^{\circ}$ due à la dérive du bateau est tout à fait normale. D'autre part, la courbe de célérité a pu varier durant la transmission la rendant encore plus critique. Pour une communication sous-marine, la connaissance à priori, précise, de la courbe de célérité du son est très utile et va décider de la profondeur d'émission.

\section{Estimation des paramètres retard, amplitude, déphasage}

Il est bien connu maintenant en acoustique sous-marine que le canal est caractérisé de manière très pragmatique par une somme de $\mathrm{N}$ trajets, par une réponse impulsionnelle sous forme de somme de Dirac, chaque trajet étant affecté d'un terme retard $\tau$ et d'une amplitude complexe: un terme d'atténuation a et un terme de phase $\phi$ (cf [1]).

$$
h(t)=\sum_{n=1}^{N} a_{n} e^{j \phi_{n}} \delta\left(t-\tau_{n}\right)
$$

Les retards et les phases sont toujours des paramètres relativement constants. Lors de l'expérimentation décrite, l'énergie arrive au même instant, avec un même retard, la variation lente de ce retard provient de la dérive du bateau émetteur due au courant. Les phases après démodulation sont difficiles à suivre (longueur.

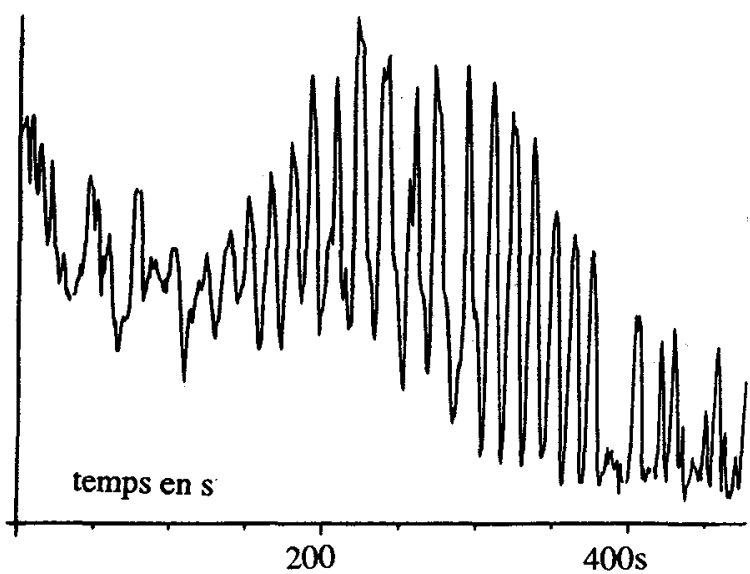

fig.2: fluctuation de l'énergie reçue à 5 miles [energy fluctuation after $5 \mathrm{~N}$-miles propagation]

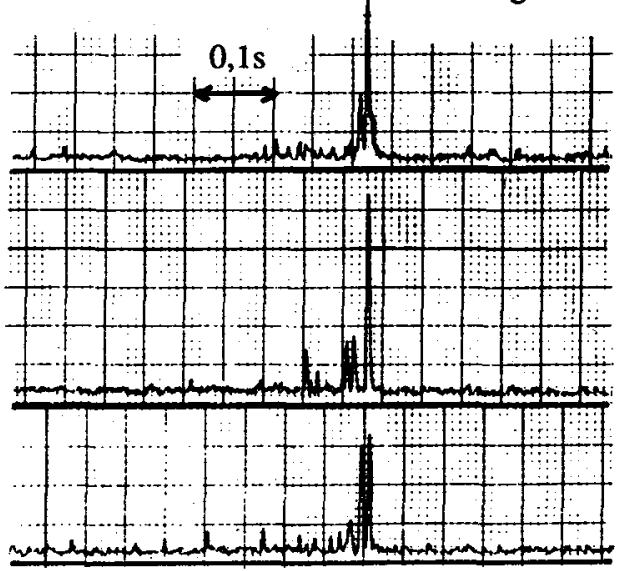

fig. 3: réponse du milieu à 47 miles [medium response after $47 \mathrm{~N}$-miles] 
d'onde $75 \mathrm{~cm}$ ). Leur mesure constitue un mesure précise des variations de distance de l'émetteur.

Il est plus difficile d'expliquer les variations d'amplitude relevées fig.2. Les séquences d'identification (pourtant de large bande: \pm 500 et $1000 \mathrm{~Hz}$ ) ne permettent pas de distinguer combien de trajets arrivent en même temps. Leur nombre est au moins de 2 car le support de la réponse obtenue est plus large que celui de l'autocorrélation d'une séquence $(2 \mathrm{~ms})$. Le tracé de rayons prévoit 2 trajets séparés de $0,7 \mathrm{~ms}$. L'utilisation de méthodes haute résolution (cf [3]) concluent à l'existence de 2 trajets également distants (les amplitudes par contre ne correspondent pas). Il semble donc que la houle, modulant différemment deux trajets très voisins, crée des interférences qui sont la cause des fluctuations quasi périodiques ( de période double \# $12 \mathrm{~s}$ ) visibles fig. 2 .

\section{TRANSMISSION LONGUE DISTANCE}

De nombreuses expérimentations de ce type ont déja été dépouillées au CEPHAG (cf [4],[5]). Nous avons effectué la transmission décrite plus haut sur une distance de 47 miles nautiques (\# $87 \mathrm{~km})$. Seule a été modifiée la fréquence porteuse $(1500 \mathrm{~Hz})$ et la profondeur de l'émetteur $(180 \mathrm{~m})$. Les résultats sont conformes à ceux trouvés antérieurement: peu de fluctuations de le fréquence $(0,1-0,3 \mathrm{~Hz})$, une énergie reçue importante (compte tenu d'une atténuation de $-5 \mathrm{~dB}$ due à la propagation) ne présentant ni évanouissements, ni modulation par la houle. On a plusieurs trajets nets de propagation: la fig. 3 représente 3 réponses obtenues simultanément sur 3 capteurs disposés verticalement à différentes profondeurs. Les retards et phases sont très stables et leurs variations représentent la dérive du bateau. Les amplitudes varient de manière plus importante que lors d'expérimentations analogues à longue distance. Ceci doit s'expliquer par le fait que l'émetteur et les récepteurs sont proches de la profondeur du chenal sonore (correspondant à la célérité minimale). Plusieurs trajets arrivent ainsi au même instant créeant ici aussi des interférences qui peuvent être sensibles. Ce phénomène n'existerait pas si l'émetteur et le récepteur n'étaient pas dans cette position (cas courant en Atlantique où le chenal est plus profond) et donc si les trajets étaient tous séparés.

\section{CONCLUSION}

Une transmission courte ou moyenne distance $(<10-15 \mathrm{~km})$ est très sensible à divers paramètres comme la position précise de l'émetteur et du récepteur vis à vis des courbes de célérité du son dans l'eau. L'étude de l'influence des variations de cette courbe sur la propagation, l'introduction de composantes statistiques dépendant des courants, de l'état de la mer et des fonds... seraient très utile pour ce type de transmission. La directivité de l'antenne émettrice, son inclinaison entrent également en jeu. Des phénomènes physiques comme la houle, les vents... vont intervenir. Tout ceci montre qu'une transmission sur une courte distance est plus délicate que sur une longue distance, essentiellement à cause d'interférences (liées aux variations de ces paramètres) modifiant de manière importante les caractéristiques de la propagation. Le cas le plus défavorable est celui des petits fonds.

Une transmission longue distance est plus simple à gèrer car, si l'énergie transmise est bien sûr plus faible, les paramètres du canal sont moins fluctuants. Le phénomène primordial est la réfraction des rayons autour de l'axe de célérité minimale (chenal sonore). Les particulatités physiques, expérimentales, aléatoires sont d'autant plus lissées que la distance est plus grande. Si la propagation s'effectue au voisinage du chenal sonore, des interférences peuvent produire également des fluctuations néfastes, rendant le milieu moins déterministe.

Ce travail a été réalisé avec le concours de la Direction des Constructions Navales.

\section{Bibliographie:}

[1] G. Loubet, J. L. Lacoume, B. Faure, F. Vial - Effet de la dispersion sur la propagation acoustique sous-marine - ler Congrès Français d'Acoustique, Lyon - 1990.

[2] G. Loubet, X. Niziers - Communication acoustique sous-marine par utilisation de codes pseudo-orthogonaux - 13 ème colloque GRETSI - Juan les Pins - Septembre 1991.

[3] V. Nimier - Contribution à l'estimation des paramètres caractérisant la propagation par trajet multiple Thèse de IINPG - CEPHAG - 1990.

[4] G. Jourdain, J.Y. Jourdain - Characterisation of submarine acoustic transmission channels - Colloque NATO ASI - Copenhagen - 1980.

[5] G. Loubet, M.A. Pallas - Caractérisation du milieu marin en tant que canal acoustique - J. Acoustique 1 (1988) pp $129-134$. 